\title{
UPS - An Ubiquitous Proximity eService for Trust Collaboration
}

\author{
Yuan-Chu Hwang ${ }^{1}$ and Soe-Tsyr Yuan ${ }^{2}$ \\ ${ }^{1}$ Department of Information Management, National United University, Taiwan \\ No. 1, Lien Da, Kung-Ching Li, Miao-Li 360, Taiwan, R.O.C. \\ yuanchu. hwang@gmail.com \\ ${ }^{2}$ Department of Management Information Systems, National Chengchi University, Taiwan \\ No. 64, Sec. 2, ZhiNan Rd., Wenshan District, Taipei City 11605, Taiwan, R.O.C. \\ yuans@mis.nccu.edu.tw
}

\begin{abstract}
Ubiquitous e-service is one of the most recent links in the chain of evolution that has characterized the different eras of the internetworking environment. The ubiquitous proximity e-service highlights the collective effort focused on collecting the user group's power as the reference for ubiquitous trust decisions. In this paper, we define the ubiquitous proximity e-service and discuss the significance of ubiquitous proximity e-service. Simulation outcomes for trust decision quality enhancement show significant improvement in all kinds of environment settings. The ubiquitous proximity e-service makes it possible for users to collaborate with the nearby user groups for establishing a reliable and trustworthy interaction environment. It also facilitates and empowers the potential benefits of various ubiquitous e-service applications.
\end{abstract}

\section{Changes of Proximity in the U-Commerce Era}

The ongoing developments of ubiquitous commerce have brought human life into a new era. Classic social science studies long ago demonstrated that proximity frequently increases the rate of individuals communicating and affiliating in organizations and communities [1,4]. Proximity also develops strong norms of solidarity and cooperation. While advanced telecommunication technologies have led some to conclude that the problem of distance has been overcome, others argue that proximity remains essential to group functioning and that new technologies cannot eliminate the challenges faced by members of geographically-dispersed teams. The essentiality of proximity may be controversial, but the definition of proximity might change owing to technological innovations in the U-Commerce era.

We proposed Ubiquitous Proximity e-Service (UPS) for exploring collective wisdom in the ubiquitous environment. The UPS highlights the collective effort focused on collecting the user group's power as the reference for ubiquitous trust decisions. Some security design was elaborated in Hwang and Yuan [10], including three conceptual methodology designs: the privacy design, reputation management design, and trust management design. But in is paper, we focus on the theoretical support of proximity value. 
The ubiquitous proximity e-service can be treated as a new scope of ubiquitous e-services that highlight the collective effort of proximity participants within a ubiquitous environment. Due to the dynamicity and complexity present in the ubiquitous world, it is unrealistic to expect humans to be able to reason and act effectively to address potential risks in the ubiquitous environment. In order to propose a new e-service paradigm that aims to mitigate potential risks and threats present in the ubiquitous e-service environment due to its flexible, dynamic, and collaborative nature, we will begin our discussion by considering the collaboration with proximal participants.

Sociologists and anthropologists have long recognized that people can feel close to distant others and develop common identities with distant others who they rarely or never meet. [2, 9] Besides geographical distance, in the U-Commerce era, proximity places increased emphasis on individual homophily personal characteristics. The principle of homophily provides the basis for numerous social interaction processes. The basic idea is simple: "people like to associate with similar others." [3, 11, 13] As mentioned above, ubiquitous proximity e-service stresses the collective efforts of participants in the dynamic environment. Homophylic user groups are more likely to combine the strength of different individuals to achieve specific objectives.

Furthermore, as stated in Metcalfe's Law: "the usefulness, or utility, of a network equals the square of the number of users." This law has been modified to consider the number and value of the network resources (i.e. available services). The Network Effect results from Metcalfe's Law, which states that, "Network effects refer to the notion that as more individuals participate in a network, the value of the network to each individual participant increases". Network adoption rate increases in proportion to network utility. Services become more valuable as more people use it, thus encouraging growing numbers of adopters.

Ubiquitous proximity e-service exploits the network effect and tries to enhance collective effort by gathering energy within a dynamic environment. The relationships within the ad-hoc ubiquitous environment involve social network theory. Interpersonal social relationships can be defined by tie strength as weak or strong ties based on the following combinations: time, emotional intensity, intimacy, and the reciprocal services which characterize the tie. [7]. According to Marsden and Campbell, tie strength depends on the quantity, quality, and frequency of knowledge exchange between actors, and can vary from weak to strong. Stronger ties are characterized by increased communication frequency and deeper, more intimate connections.

Although strong ties tend to provide greater social support than weak ties (emotional aid, goods and services, companionship, and a sense of belonging), weak ties tend to link individuals to other social worlds, providing new sources of information and other resources.[8] Their very weakness means that they tend to connect people who are more socially dissimilar than those connected via strong ties. Individuals with few weak ties within a community become isolated from receiving new information from outside circles and can only hear information re-circulated within their own clique of close friends [8]. A weak tie link linking strongly tied groups is termed a local bridge [7]. Weak ties contribute to social solidarity; community cohesion increases with the number of local bridges in a community [7]. 
According to Friedkin [5] the mix of weak and strong ties increases the probability of information exchange.

The ubiquitous e-service environments are in an ad-hoc composition where social relationships may not well spread. Strong ties may only rarely be available within this ad-hoc environment. The nature of ad-hoc e-service is such that rare connections between individuals are more likely to establish. Proximity may be the reason for participants establishing ties. Even weakness ties then have an opportunity to become strengthened interpersonal relationships.

Homophily (namely, love of the same) describes the tendency of individuals to associate and bond with similar others. Homophily has been found in numerous network studies. By highlighting the homophily of e-service participants, these isolated individuals can be treated as a group with proximity (that is: common goals, similar interests, etc.). Interpersonal ties can be established in addition to some interactions. Loose-coupled e-service participants thus can be empowered to form groups/clusters with weak ties. Proximity thus enables ad-hoc e-service participants to contribute their strength for ubiquitous collective wisdom.

\section{Significance of Ubiquitous Proximity e-Service}

The main value of ubiquitous proximity e-service utilizes the network effect from the collective effort of interpersonal social network. By obtaining unique, nonreproducible interpersonal experiences from e-service environments, those information sources originate and shape collective wisdom. The involvement of more participants further increases the possibility of strengthening collective wisdom. The collective value derives from individual mental proximity. The characteristics of proximity encourage loose-coupled or isolated individuals to form groups with weak tie relationships and facilitate the creation of collective wisdom. Information diffusion and gathering via the Peer-to-Peer method can obtain "unique" data sources. The following paragraphs discuss collective wisdom based on the proximity e-service environment and a critical trust issue regarding how to collaborate with unfamiliar strangers.

Collective wisdom has a similar meaning to the term "collective intelligence", which describes intelligence based on the collaboration and competition of numerous individuals (an intelligence that appears to have a mind of its own). One pioneer of research on collective intelligence, George Pór, defined collective intelligence as: "the capacity of a human community to evolve toward higher order complexity thought, problem-solving and integration through collaboration and innovation."[6]

The collective wisdom regarding ubiquitous proximity e-service is based on social networks. Since ubiquitous proximity e-services may utilize Internet environments, they transmit information various ways: (1) the external method that permits effective information spread and diffusion; (2) the internal method that helps individuals to gather and obtain useful information via personal social networks. Proximity e-service participants propagate information voluntarily via their own social networks voluntarily. Information diffusion for proximity e-services is more efficient than in Internet environments, and weak ties help information propagation and diffusion via the personal networks and relationships of nearby users. 
However, a critical problem exists regarding trust decisions for strangers, "Why individuals should share information with strangers in an unfamiliar environment?" This problem involves problems of both interpersonal trust and efficiency. Relying solely on fixed Internet it is impossible to establish such extensive interpersonal trust networks in an ad-hoc e-service environment. In the ad-hoc e-service environment, it is necessary to integrate social networks with trust issues. It can be said that ubiquitous e-service participants may also be unfamiliar with each other. However, in the real world, it is usually accepted that "trust" or "confidence" is necessary for commerce activities. Despite the need for precautions, it is said that: "you have to trust your partner". The commerce environment is rife with asymmetric information, moral hazard, opportunism, and so on. Vulnerability exists in commerce environments. Nevertheless, collaboration may be necessary in many situations in which goals cannot be achieved by single units (persons, firms, groups etc.). Thus, adhoc e-service participants have to accept, at least, a minimum vulnerability to achieve cooperation with partners. An optimistic concept may increase the chance of collaboration becoming a reality. However, cautious assessments of interaction events are also crucial.

It is difficult for users to collaborate with complete strangers. No collective wisdom can be established in environments in which participants are completely isolated. A significant value of the proximity e-service lies in the increased possibility of establishing innovative social network relationships. From the interpersonal perspective, unfamiliar strangers can make connections with individuals who are proximal and homoplastic to him (that is, shared interests cause users to gather at a single exhibition). The strength of proximity gives people better chances to make interpersonal connections, including both weak ties (i.e. someone you know each other) and strong ties (i.e. good friends).

Similarity breeds connections. [12] Ubiquitous proximity e-service utilizes homophily to connect separated individuals via weak tie relationships. These weak connected groups together with their original owned interpersonal connections then can enhance the collective effort by extensive information sharing and cooperation. By combining those interpersonal tie relationships proximity e-services can more easily cause information diffusion and effectively encourage collective wisdom.

\section{Justification of Theoretical Support}

The concept of the small-world phenomenon was observed over 30 years ago in social systems. The small world phenomenon (also known as the small world effect) is the hypothesis that everyone in the world can be reached via a short chain of mutual acquaintances. Small-world behavior can be defined generally and physically by considering the efficiency of information exchange over the network. The proximity value benefits from the small world phenomenon, which gathers available information sources and facilitates collaboration within the ubiquitous e-service environment. There are two aspects of the justification of theoretical support for proximity value: Efficiency and Cost. 
Since we have redefined the proximity in ubiquitous e-service environment. We will compare the value of ubiquitous proximity e-service with the Internet e-service environment. Comparisons are made between two scenarios: ZigBee-based ubiquitous proximity e-service environment and Internet-based WiMax e-service environment.

First, this study compares the chance to establish weak-tie connectivity for collective wisdom. Ubiquitous proximity e-service focuses on the characteristic of "Homophily". As mentioned above: (1) People like to associate with similar others, (2) Similarity begets friendship, (3) People love those who are like themselves. Proximity e-service in ubiquitous environment is characterized by "geographical proximity" as well as "user characteristic proximity". Homophily leads unfamiliar participants to form new connections. Participants may have the opportunity to make connections via weak-tie relationships, and may even have the opportunity to form interest groups. ZigBee supports the local range connections and thus is suitable for proximity e-service, which also emphasis on interacting with nearby participants. Compared to individuals who only have the "user characteristics" homophily, ubiquitous proximity e-service based on ZigBee has more opportunities for unfamiliar individuals to build some new relationships. (Since proximity e-service own both user characteristics homophily and geographic homophily). Proximity increases the opportunities for unfamiliar ubiquitous e-service participants to form weak-tie interpersonal relationships.

Internet-based WiMax makes it more convenient for mobile users to connect to the world. Although users may have the characteristic proximity in the internet-based WiMax environment, their geographic distances are significantly larger than in the ZigBee-based environment. (9.6 kilometers for WiMax versus 100 meter for ZigBee) Extensive service range is not the advantages for real-time interactions particularly given that e-service focuses on communicating with nearby participants. Since individuals may sometimes wish to make connection with other distant individuals. It is unrealistic to establish real-time interactions over long distances. According to Hill and Dunbar [15], social networks have a cap of approximately 150 individuals (above this size they cease to be effective). Increasing connection numbers by extending service range cannot improve social network efficiency. Furthermore, the homophily characteristics of broad range peer groups are looser than the surrounding proximal users in terms of network density. It is less efficient for individuals to perform realtime interactions or obtain e-services from service providers. The effect that weak-tie gathered from user characteristics homophily also exists in the internet-based WiMax environment. However, in the absence of geographical homophily, WiMax is weaker than the proximal range of ZigBee-based ubiquitous proximity e-service. In terms of the intensity of real-time interactions, ZigBee-based ubiquitous proximity e-service is stronger than Internet-based e-services.

Hereby, we have the first explanation for proximity e-service in ubiquitous environment as follows: "Ubiquitous proximity e-service has better opportunities to build up weak-tie connectivity than internet e-service environment."

$$
\rho_{\text {proximity }}>\rho_{\text {Internet }}
$$

where $\rho$ represents the probability of establishing interpersonal connections. 
Second, this study compares the cost of establishing social relationships. The cost of establishing the social network can be various, including communications costs, the involvement of the social network, etc. All of these costs are related to communications between mobile devices. Transmissions increase with involvement of virtual sociality. However, increased communications generally result in increased power consumption for transmitting messages via mobile networks. Without loss of generality, this investigation uses power consumption as the communication cost which represents the cost of building interpersonal relationships. Indeed, mobile devices suffer power supply constraints. Without power supply, mobile users are isolated from their networks and their mobile device becomes useless. As mentioned above, ZigBee describes a standardized wireless protocol for personal area networking, or "WPAN". ZigBee differs from other wireless standards in being designed to serve a diverse market of applications requiring low cost and low power wireless connectivity, and provides greater sophistication than was previously available for the price. ZigBee focuses on low data rate and low duty cycle connectivity, a segment that existing standards to not service well. WiMax supports wide range communications, but has higher power consumption than ZigBee, and thus requires higher capacity battery. According to Texas Instruments (TI), WiMax currently stays in the stage for mobile users accessing the broadband network. The mobility of WiMax will confront with roaming problem, power consumptions problems, network switching issues, etc. in the future. Attempts to apply WiMax to mobile applications will meet these problems.

This study provides another explanation for proximity e-service in ubiquitous environments, as follows: "ZigBee-based proximity e-service can establish social relationships more cheaply than Internet e-service environments."

$$
\gamma_{\text {proximity }}<\gamma_{\text {Internet }}
$$

$\gamma$ represents the establishment cost for establishing interpersonal connections.

Information diffusion via proximity e-service environment. Unlike the fixed internetbased network topology, mobile ad hoc networks (MANETs) comprise mobile devices fitted with short range radio transmission. Devices can communicate within their respective radio ranges. The mobility of ubiquitous e-service participants leads to frequent topology changes in ubiquitous proximity e-service environment. Considering information diffusion efficiency within ubiquitous proximity e-service environments, this study found that traditional information propagation theory may not be applicable to current ubiquitous e-service environments. Ubiquitous proximity e-service benefits from social network theory and improves the diffusion efficiency through various tie relationships.

Based on the definition of economic small world, the economic small world focuses on the low cost and high efficiency of information propagation. Consider the information propagation between two nodes $\mathrm{i}$ and $\mathrm{j}$. The path length (that is, distance) between the two nodes is defined as $\left\{\mathrm{d}_{\mathrm{ij}}\right\}$. Moreover, the information diffusion Efficiency $(\varepsilon)$ is defined as $1 /\left\{\mathrm{d}_{\mathrm{ij}}\right\}$. When distance $\left\{\mathrm{d}_{\mathrm{ij}}\right\}=+\infty$, Efficiency $(\varepsilon)=0$.

Ubiquitous proximity e-service is based on mobile ad-hoc network structure, in which users are generally unfamiliar with surrounding participant peers. In the absence of any existing relationships, those total strangers may not establish 
connections. If Peer $_{i}$ and Peer $_{j}$ are totally unfamiliar with each other then no relationships exist between the two peers. Since the peers are unconnected, the path length (i) to $(\mathrm{j})$ is $+\infty$, meaning $\left\{\mathrm{d}_{\mathrm{ij}}\right\}=+\infty$. Based on the efficiency definition, the Information Diffusion Efficiency is zero. $(\varepsilon=0)$

Two of the ideas mentioned above are: (1) Ubiquitous proximity e-service has a better chance of improving weak-tie connectivity than Internet e-service environment. (2) ZigBee-based ubiquitous proximity e-service has lower cost for establishing social relationships than Internet e-service environment.

Ad-hoc e-service environment's nature (i.e. short term lived identities) may cause participant unfamiliarity. In such e-service environment, proximity e-service has a better chance of establishing weak-tie connections with nearby peers while achieving lower communication costs.

Because $\varepsilon$ is directly proportional to $\rho$. Additionally, $\varepsilon$ is inversely proportional to $\gamma$. Accordingly, it was found that $\varepsilon=\beta \quad(\rho / \gamma)$. Efficiency $(\varepsilon)$ increases with increasing $\rho$. Higher $\operatorname{Cost}(\gamma)$ also leads to lower Efficiency $(\varepsilon)$. In this formula, $\beta$ is a constant greater than 1 .

From (1) and (2), since $\rho_{\text {proximity }}>\rho_{\text {Internet }}$ and $\gamma_{\text {proximity }}<\gamma_{\text {Internet }}$, it is concluded that $\varepsilon_{\text {proximity }}>\varepsilon_{\text {Internet }}$

$$
\varepsilon_{\text {proximity }}>\varepsilon_{\text {Internet }}
$$

Some possible future scenarios are considered below:

(a) The service range of the Internet-based WiMax e-service environment is reduced, reducing in a situation where $\rho_{\text {proximity }} \geqq \rho_{\text {Internet }}$. Since $\gamma_{\text {proximity }}<\gamma_{\text {Internet }}$, in which case the information diffusion efficiency of ubiquitous proximity e-service remains higher than in the Internet-based WiMax environment.

(b) Advanced technologies reduce communication costs, leading to $\gamma_{\text {proximity }} \leqq$

$\gamma_{\text {Internet }}$. Since $\rho_{\text {proximity }}<\rho_{\text {Internet }}$, in which case the information diffusion efficiency of ubiquitous proximity e-service remains higher than in the internet-based WiMax environment.

Neither of these situations influences the result. This study thus reaches the following conclusion: The Information Diffusion Efficiency in the ubiquitous proximity e-service environment exceeds that in the Internet-based environment.

\section{Value of Proximity: A Case Scenario}

Participant social relationships of a conference scenario resemble a small world network. Small world networks have shorter average path length between nodes and higher node clustering than in the case for a random distribution. Watts and Strogatz [14] demonstrate how social networks, electricity power grids and neural networks small world properties and how their model can be used to create graphs with such properties. The conference attendees inhabit a proximity e-service environment, which participants are in some level similarity of their interests in geographic proximity as well as cognitive proximity. Figure 1 represents the conference proximity e-service scenario. 
This study considers the following scenario that offers proximity e-service in a conference. The scenario involves a conference with numerous delegates, at which several sessions generally occur simultaneously. Attendees moving freely between sessions, depending on the subject and their interests. Relationships between colleagues and friends are considered strong-tie relationships. Meanwhile, familiar strangers describe individuals who are regularly encountered but with whom there are no interactions. Some strangers encountered repeatedly at the same conferences over consecutive days may be treated as familiar stranger during a later meeting in a restaurant. Familiar strangers can have different varieties of proximity to a given individual, with geographic proximity and cognitive proximity (interest in similar research topics) representing the possibility for strangers to form weak relationships with others.

\section{Proximity implies lower communication cost and higher opport unity to establish weak-tie connections}

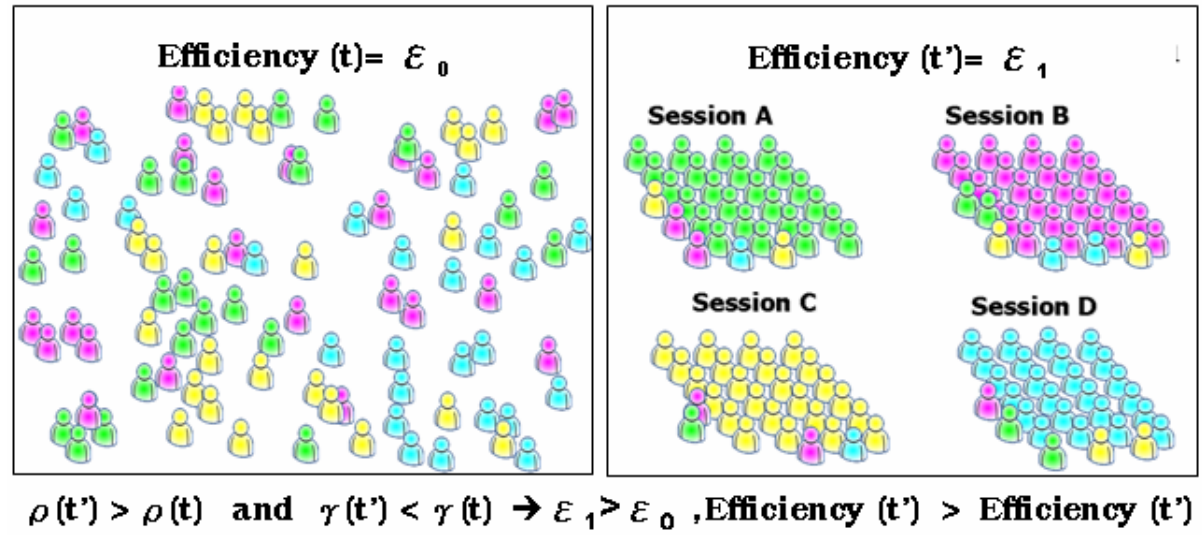

Fig. 1. Conference proximity e-service scenario

Assuming a proximity e-service environment is available in the conference, each participant has their own mobile device with facilities for exchanging information with others within a limited range. Individual mobile users manage their own information, including owner identity, scheduled presentations, copies of presentation slides, bibliographic references and URLs. Users can also provide research interest information and a list of colleagues/friends who are also attending the conference.

Mobile users can exchange information dynamically and spontaneously given physical proximity. In such contexts, individual physical encounters provide good opportunities for information exchange. Such information exchange can occur only during a limited period which can not be statically known. Owing to the limited communication time available during each encounter, it is important to rapidly access the most relevant information.

Some forms of collective wisdom can be generated. The group notes provide an example: all participants can take notes of their own or share notes with others. (For example, sharing notes with colleagues attending another session) During discussion 
of conference sessions, participants can contribute their ideas for discussion by referring to their conference notes. Moreover, presenters can update their slides and make notes during discussions and share them with session participants. These personal notes can then be gathered in the form of group notes containing numerous heterogeneous viewpoints. These group notes may lead to extensive discussion regarding the social networks of individual participants simultaneously and participants can contributes some innovative thoughts. Colleagues and friends have similar interests particularly background domain. Those proximal participants may form strong tie clusters in the conference social network. The sessions include numerous network relationship clusters. The proximity of "cognitive view" (that is, participant homophily) may form weak tie relationships which will increase the effectiveness of information diffusion. The characteristics of proximity make it possible for those total strangers to treat others as familiar strangers and generate some collective efforts. Furthermore, collective wisdom is more likely to occur in a proximity e-service environment. Conference attendees may find some clues to connect to some unfamiliar people within the proximity e-service environment and carry out extensive collaboration.

Another form of collective wisdom is considered below. Andrew has just arrived at the conference. While registering at the conference, Andrew runs into his old friend and ex-colleague, Katrina, who is on her way to the same conference. They start talking about the research they are working on and decide that they would like to try to write a paper for another conference with a deadline in a few weeks. To plan their writing, they exchange current contact information, information about the conference, notes, documents, work pointers, and so on. This information is located on one or other of their mobile devices. Even though only a subset of these resources would normally be available to each other, they can still share the information they need for their collaboration.

Another example involves Katrina planning to publish a paper in another domain with which Andrew is not familiar. However, Andrew is familiar with an editor-inchief who is also interested in this domain. Andrew thus sends an email to the editor and recommends Katrina's work, and the editor then invites Katrina to join a research discussion. This relationship connects the isolated personal social networks and binds the strong tied relationship networks of individuals.

However, in proximity e-services all interactions occur within the context awareness environment. Contextual awareness means that private mobile user information may be exposed to others. In certain cases the anonymity of the owner of the information must be guaranteed, for example during an encounter between individuals who have previously never met and who do not want to know each other. In other situations the confidentiality of information to be transferred must be guaranteed.

The collective wisdom of ubiquitous proximity e-service environment comprises various levels. In ad-hoc ubiquitous e-service environments, even participants are not familiar with each other. UPS enables proximal e-service participants to contribute their experiences and form collective wisdom. Based on user preference or behavioral stereotype settings, UPS facilitates collective filtering which provides information to 
identify trustworthy partners via an experience co-creation process. In such unfamiliar ad-hoc environments it is good to have information to serve as a reference for making good decisions.

Those ubiquitous proximity e-service cases have some common attributes that each participant just owns some pieces of information. Aggregate more information pieces will improve the decision quality, and then the collective wisdom will appear. Mobile and ubiquitous computing has changed its emphasis from "Anywhere, anytime" into "In this specific place, at this specific moment, for this specific person", and pervasive communication is connecting proximal e-service participants to contribute ubiquitous collective wisdom.

\section{Simulation Justification}

The ubiquitous proximity e-service is designed to enhance the decision quality on trust evaluation via exploration of the collective wisdom of the surrounding user groups. In the ad-hoc e-service environment, a multitude of transactions take place between anonymous sellers and buyers. Since users do not have permanent identities, they have to handle the trust problem. Mostly, a seller deals with this problem by insisting on payment in advance, thereby, protecting himself from deceitful buyers. The seller delivers the service package only after receiving payment from the buyer. The buyer therefore must be confident of the seller's willingness to deliver the service package.

Because the available e-service provision is highly dependent on the resources of the service provider, customers may not always get what they ask for. Aggressive sellers who are not able to provide requested services may decide to promote alternative choices, but customers may not want to waste their computational resources on annoying spam messages. After receiving the seller's response, buyers have to decide whether to accept the provided choice. The interaction between the buyer and the seller can be formalized as a simple trust game.

The goal of simulation is to verify whether the collective wisdom gathered from ubiquitous environment could improve the decision quality for estimating the trustworthiness of unfamiliar user.

Healthy Environment vs. Malicious Environment. Lack of trustworthy infrastructure within the ad-hoc mobile e-service environment, each peer basically needs to maintain all threats in the environment on its own. Ubiquitous proximity e-service enables the collective wisdom from e-service participants and supports collaborative trust evaluation of nearby users. It should be expected that UPS could integrate available resources within an e-service environment to prevent malicious events. In order to evaluate the performance of UPS in various situations, we then set up three kinds of environments: Healthy Environment, Malicious Environment, and Neutral Environment. The "Healthy Environment" contains $80 \%$ honest users and 20\% malicious users. On the contrast, the "Malicious Environment" contains 80\% malicious users and $20 \%$ honest users. A "Neutral Environment" has half honest users and half malicious users as a benchmark for normal environment settings. We stabilize other parameter settings: there are 20 users within the e-service environment 
with a balanced buyer-to-seller ratio. User behaviors are assumed in normal distribution within a normal resource level.

Figure 2 illustrates that use of UPS would help decrease cheat transaction rate. As interaction increases, the collective wisdom generated from participants' coexperience would assist deter cheat transactions. The use of UPS is most effective in malicious environment - the cheat transaction rate could be lowered to $29 \%$ initially and would be down to $14 \%$ after 100 transactions take place. As in the "Healthy Environment", with the use of UPS, cheat transaction rate would remain under $8 \%$ for the whole simulations and drop to $1 \%$ after 100 transactions take place. Simulation results indicate UPS's collective wisdom mechanism would assist improve decisionmaking quality for all environment settings.

Cheat Transaction Rate

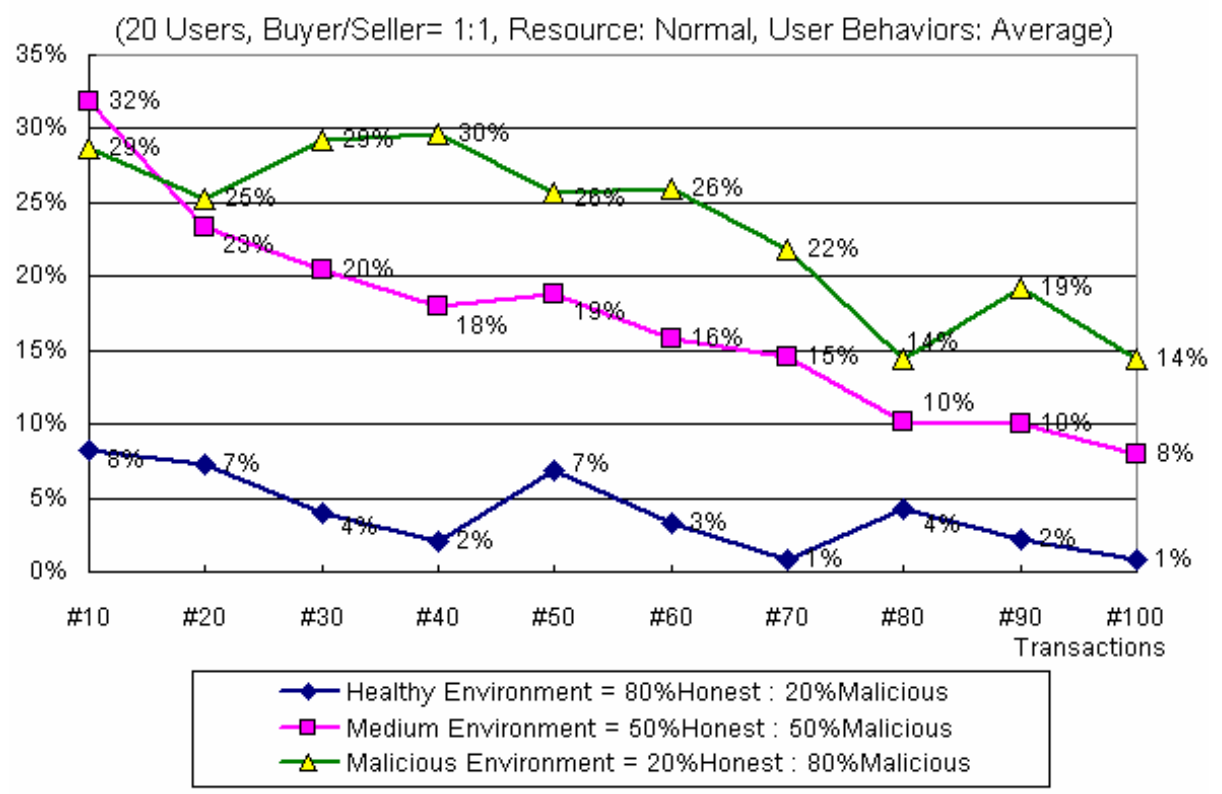

Fig. 2. Cheat transaction rate in Healthy/ Neutral /Malicious Environment

The simulation results clearly show that ubiquitous proximity e-service makes it possible for users to collaborate with the nearby user groups for establishing a reliable and trustworthy interaction environment. The ubiquitous proximity e-service realizes the collective wisdom and provides a feasible solution for quality decisions in the dynamic and distributed environment.

\section{Conclusion}

Ubiquitous e-service is one of the most recent links in the chain of evolution that has characterized the different eras of the internetworking environment. In order to leap 
the trust barrier for the user to embrace these ubiquitous e-services, we present a ubiquitous proximity e-service for exploring collective wisdom in the ad-hoc ubiquitous environment. Simulation outcomes for trust decision quality enhancement show significant improvement in all kinds of environment settings. The ubiquitous proximity e-service makes it possible for users to collaborate with the nearby user groups for establishing a reliable and trustworthy interaction environment. It also facilitates and empowers the potential benefits of various ubiquitous e-service applications.

\section{References}

1. Allen, T.J.: Managing the Flow of Technology. MIT Press, Cambridge, MA (1977)

2. Anderson, B.: Imagined Communities. Verso, London (1983)

3. Aristotle: The Nicomachean Ethics. H. Rackham, translator. Harvard University Press, Cambridge, MA (1934)

4. Festinger, L., Schachter, S., Back, S.: Social Pressures in Informal Groups: A Study of Human Factors in Housing. Stanford University Press, Palo Alto, CA (1950)

5. Friedkin, N.E.: Information flow through strong and weak ties in intraorganizational social networks. Social Networks 3, 273-285 (1982)

6. Pór, G.: The Quest for Collective Intelligence. In: Gozdz, Kazmierz (eds.) Community Building: Renewing Spirit \& Learning in Business, New Leaders Press, San Francisco (1995)

7. Granovetter, M.: The Strength of Weak Ties. American Journal of Sociology 78, 1360 1380 (1973)

8. Granovetter, M.: The strength of weak ties: A network theory revisited. In: Marsden, P.V., Lin, N. (eds.) Social Structure and Network Analysis, pp. 105-130. Sage, Beverly Hills, CA (1982)

9. Habermas, J.: The structural transformation of the public sphere. MIT Press, Cambridge, MA (1991)

10. Hwang, Y.C., Yuan, S.T.: A Privacy-Aware Identity Design for Exploring Ubiquitous Collaborative Wisdom. LNCS, vol. 4490, pp. 433-440. Springer, Heidelberg (2007)

11. Lazarsfeld, P., Merton, R.K.: Friendship as a social Process: A Substantive and Methodological Analysis. In: Berger, M., Abel, T., Page, C.H. (eds.) Freedom and Control in Modern Society, pp. 18-66. Van Nostrand, New York (1954)

12. McPherson, M., Smith-Lovin, L., Cook, J.: Birds of a feather: Homophily in Social Networks. Annual Review of Sociology 27, 415-444 (2001)

13. Plato. Laws. Plato in twelve volumes, Bury translator, vol. 11, p. 837. Harvard U. Press, Cambridge (1968)

14. Watts, D., Strogatz, S.: Collective Dynamics of Small-World Networks. Letters to Nature 393, 440-442 (1998)

15. Hill, R.A., Dunbar, R.I M.: Social network size in humans. Human Nature 14(1), 53-72 (2003) 